\title{
A Spectrophotometric Atlas of White Dwarfs Compiled from the IUE Archives
}

\author{
Steven R. Swanson (Dartmouth College, U.S.A.) \\ Gary Wegner (Dartmouth College, U.S.A.)
}

\section{INTRODUCT ION}

In the past ten years, more than 775 low resolution spectra of white dwarfs have been taken with the International Ultraviolet Explorer satellite (IUE). This wealth of information has yielded many new discoveries in the field of white dwarf research; a few of which include: the $\lambda 1400$ and $\lambda 1600$ quasi-molecular features discovered in hydrogen rich DA white dwarfs (Greenstein 1980; Wegner 1982, 1984; Nelan and Wegner 1985; and Koester et. al. 1985), strong C I lines in some DQ white dwarfs (Koester, Weidemann, and Vauclair 1980; Wegner $1981 \mathrm{a}, \mathrm{b})$, and the absence of these same lines in hotter DB white dwarfs by Wegner and Nelan (1987) which may indicate convective mixing (Pelletier et al. 1986).

In this study, spectra from the IUE archives will be re-processed and corrected for changes which have occured in data reduction procedures over the past ten years. For example, all SWP (short wavelength primary) spectra processed before November 4, 1980 at Goddard Space Flight Center (GSFC) and March 10,1981 at VILSPA, used an intensity transfer function (ITF) which was in error. This resulted in reduced absolute fluxes. Another major correction is due to the gradual degradation of the LWR (long wavelength redundant) camera over time, but which was not noticed until after many spectra were affected. Therefore, in order to create a relatively homogeneous set of data, reprocessing of most of the spectra is necessary. Of the 775 spectra which will be used in this study, there are 528 spectra of DA white dwarfs ( 354 of those are SWP), 195 spectra of DB white dwarfs (117 of those are $S W P$ ), and 51 spectra of other types of white dwarfs which include DZ and DQ types. These spectra comprise approximately 200 different stars. One of the results of this study will be the detection of the spectra which have been misclassified in the IUE merged log. Once these spectra have been reprocessed, an atlas will be published of the resultant merged spectra as well as tables of the flux values.

This large database will then be used to do a statistical study on the mass distribution of the white dwarfs and also their luminosity function. Several studies on these topics have been done using visual data only (Koester, Schulz, and Weidemann 1979; Shipman 1979; and Fleming, Green, and Liebert 1986). The mass distribution and the luminosity function of white dwarfs are very strongly tied to theories concerning the origin and cooling of white dwarfs, and many detailed calculations have been done to predict these properties (e.g. Iben and Tutukov 1986). By using a model atmosphere program to study the UV spectral energy distribution, the effective temperature ( $\left.\mathrm{T}_{\text {eff }}\right)$ and the surface gravity $(\log (\mathrm{g}))$ can be obtained. When these parameters are combined with trigonometric parallaxes, values of the radii can be obtained. Currently, aproximately 57 of the white dwarfs in the archives have trigonometric parallaxes.

\section{DATA ANALYSIS}

The first step in the reprocessing of the archived data is to examine the quality of the data, and reject very noisy spectra. Then the remaining data are re-extracted from the two- 

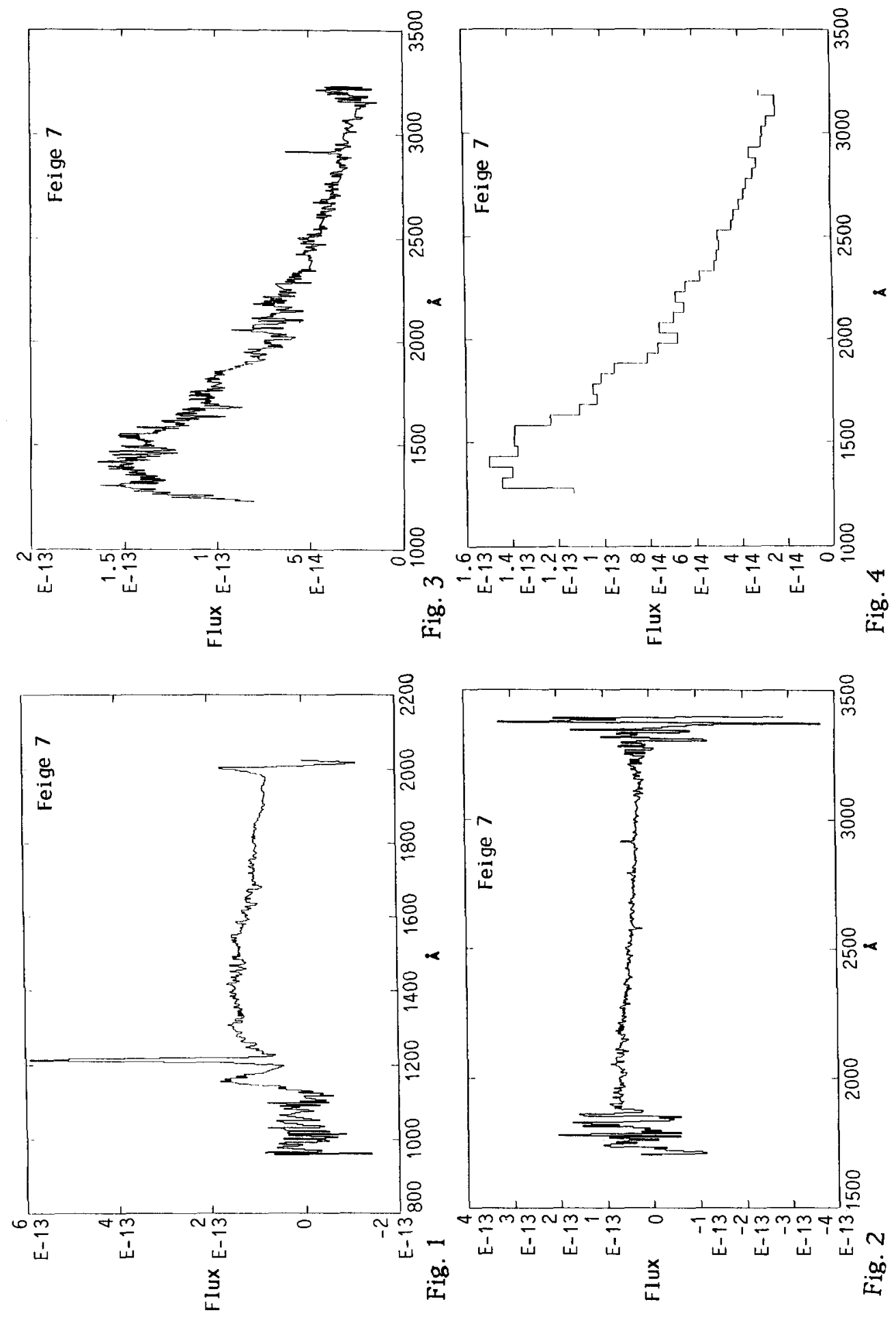
dimensional data files. The re-extraction is necessary in order to apply the ITF correction for early SWP spectra and the LWR camera degradation correction. In this way, the finished spectra will be a homogeneously reduced database. The extraction of the onedimensional spectra will be done using one of two techniques. The first uses a rectangular extraction slit, which is most accurate for the high signal to noise spectra. The rectangular method is the standard extraction technique for IUE spectra, and is the fastest method to use. The second method is a gaussian slit extraction. This is more time consuming, but results in more accurate fluxes for for lower quality data.

The one-dimensional spectra are then corrected for cosmic ray hits, blemishes, and reseau calibration points. Figures 1 and 2 show typical SWP and LWR spectra respectively, before the noisy ends have been trimmed off. Next all exposures of the same star are summed for each wavelength region and the resultant $S W$ and $L W$ spectra are merged, giving an effective spectral range of about $1200 \AA$ to $3400 \AA$ (see Fig. 3 ). The final step in the re-reduction will be to bin these spectra in $50 \mathrm{~A}$ bins for the final atlas (Fig. 4 shows an example of a final atlas spectrum).

When the above study has been finished, the model atmosphere program LUCIFER (Nelan 1985. McMahan 1986) will be used to analyse the UV spectra (and combined optical spectra where available). With this program $T_{\text {eff }}$ and $\log (g)$ will be determined for each star.

\section{CONCLUSION}

This study will result in a homogeneous photometric set of ultraviolet energy distributions for a large number of white dwarfs. The resulting atlas should prove to be very useful to the astronomical community. Also the statistical study of the mass distribution and the luminosity function of white dwarfs using this ultraviolet data will be a valuable compliment to the similar studies which have been done in the optical wavelengths.

\section{REFERENCES}

Auer, L. H. and Mihalas, D. 1970, M.N., 149,65.

Fleming, T., Liebert, J., and Green, R. F. 1986, Ap. J., 308, 176.

Greenstein, J. L. 1980, Ap. J. (Letters), 241, L89.

Grenfell, T. C. 1974, Astr. Ap., 31, 303.

Iben, I. and Tutukov, A. V., 1986, Ap. J., $311,753$.

Koester, D., Weidemann, V., Zeidler-K.T., E.-M., and Vauclair, V. 1985, Astr. Ap., 142 , L5.

Koester, D., Weidemann, V., and Vauclair, G. 1980, Astr. Ap., 83, L13.

Koester, D., Schulz, H., and Weidemann, V. 1979, Astr. Ap., 76. 262.

Liebert, J., Wesemael, F., Hansen, C. J., Fontaine, G., Shipman, H. L., Sion, E. M., Winget, D. E., and Green, R. F. 1986, Ap. J., 309,

McMahan, R. K. 1986, Ph.D. Thesis, Dartmouth College.

Nelan, E. P. 1985, Ph.D. Thesis, Dartmouth College.

Nelan, E. P., and Wegner, G. 1985, Ap. J. (Letters), 289, L31.

Pelletier, C., Fontaine, G., Wesemael, F., Michaud, G., and Wegner, G. 1986, Ap. J., 307, 242.

Shipman, H. L. 1979, Ap. J., 228, 240.

Sion, E. M. 1986, P.A.S.P., $98,821$.

Wegner, G. $1981 \mathrm{a}$, Ap. J. (Letters), 245, L27.

Wegner, G. $1981 \mathrm{~b}$, Ap. J. (Letters), 248, L129.

Wegner, G. 1982, Ap.J. (Letters), 261, L87.

Wegner, G. 1984, A. J., 89, 1050.

Wegner, G. and Nelan, E. P. $1987, A p . J$., in press.

Zeidler-K. T., E.-M., Weidemann, V., and Koester, D. 1986, Astr. Ap., $155,356$. 